\title{
Frameshift events predict anti-PD-1/L1 response in head and neck cancer
}

\author{
Glenn J. Hanna, ${ }^{1}$ Patrick Lizotte, ${ }^{1,2}$ Megan Cavanaugh, ${ }^{1,2}$ Frank C. Kuo, ${ }^{3}$ Priyanka Shivdasani, ${ }^{3}$ \\ Alexander Frieden, ${ }^{3}$ Nicole G. Chau, ${ }^{1}$ Jonathan D. Schoenfeld, ${ }^{4}$ Jochen H. Lorch, ${ }^{1}$ Ravindra Uppaluri, ${ }^{5}$ \\ Laura E. MacConaill, ${ }^{3,6}$ and Robert I. Haddad' \\ 'Department of Medical Oncology, and ${ }^{2}$ Robert and Renee Belfer Center for Applied Cancer Science, Dana-Farber \\ Cancer Institute, Boston, Massachusetts, USA. ${ }^{3}$ Department of Pathology, Brigham and Women's Hospital, Boston, \\ Massachusetts, USA. ${ }^{4}$ Department of Radiation Oncology, and ${ }^{5}$ Department of Head and Neck Surgical Oncology, \\ Dana-Farber Cancer Institute, Brigham \& Women's Hospital, Boston, Massachusetts, USA. ${ }^{6}$ Center for Cancer Genome \\ Discovery, Dana-Farber Cancer Institute, Boston, Massachusetts, USA.
}

Programmed cell death protein 1 (PD-1) inhibitors have efficacy in treating squamous cell carcinoma of the head and neck (SCCHN), but objective response rates are low. PD-1 ligand (PD-L1) expression alone is not considered a robust predictor of response and additional biomarkers are needed. This 3-year observational cohort followed 126 SCCHN patients treated with anti-PD-1/L1 therapy. Prior to treatment, 81 (64\%) had targeted massively parallel tumor sequencing. Of these, 42 (52\%) underwent fluorescence-activated cell sorting and PD-L1 immunohistochemistry for tumor immunoprofiling. Six (5\%) complete responses (CRs) and 11 (9\%) partial responses (PRs) were observed. Those treated with prior chemotherapy $(98,78 \%)$ versus only surgery and/or radiation had longer overall survival (OS) (10 vs. 3 months, $P=0.02$ ). Smokers had a higher total mutational burden (TMB) $(P=0.01)$. Virus-positive patients had a lower TMB $(P<0.01)$ and improved OS $(P=$ 0.02 ). Among virus-negative responders, NOTCH1 and SMARCA4 were more frequently mutated and frameshift events in tumor suppressor genes occurred more frequently $(P=0.03)$. Higher TMB and CD8 ${ }^{+}$T cell infiltrates predicted anti-PD-1/L1 benefit $(P<0.01, P<0.01$, respectively) among virus-negative tumors. TIM-3/LAG-3 coexpression with $P D-1$ was higher on $\mathrm{T}$ cells among nonresponders $(P=0.03$ and 0.02 , respectively). Somatic frameshift events in tumor suppressor genes and higher TMB among virus-negative SCCHN tumors predict anti-PD-1/L1 response.

Authorship note: LEM and RIH are co-senior authors and contributed equally to this work.

Conflict of interest: $\mathrm{CJ}$ H receives institutional research support from BMS and EMD Serono. JDS serves on the scientific advisory board for BMS, Debiopharm, Nanobiotix, and Astra Zeneca. JHL receives institutional research support from Bayer, BMS, Novartis, and Millennium. RIH receives institutional support and consults for BMS, Merck, Astra Zeneca, Pfizer, and Celgene in addition to consulting for Eisai and Cenzyme, and is also a member of the National Comprehensive Cancer Network head and neck cancer panel. RU is on the scientific advisory board at Merck.

Submitted: November 21, 2017 Accepted: January 11, 2018 Published: February 22, 2018

Reference information: JCI Insight. 2018;3(4):e98811. https:// doi.org/10.1172/jci.insight.98811.

\section{Introduction}

Immune checkpoint ligands serve to mitigate normal immune cell signaling, and represent a well-described component of cancer immune evasion (1). Therapies that target checkpoint receptors such as programmed cell death protein 1 (PD-1) and its ligand (PD-L1) have proven clinical benefit across multiple cancer types, including squamous cell carcinoma of the head and neck (SCCHN). Monoclonal antibodies that block PD-1 are approved for patients with advanced, platinum-refractory SCCHN, but overall response rates fail to exceed $20 \%$ in unselected patients (2-4). While early studies suggest that patients with human papillomavirus-associated (HPV-associated) disease and those with detectable PD-L1 expression derive greater benefit (1-3), these findings alone currently do not guide patient selection.

Beyond the PD-1/L1 checkpoint axis, efforts to understand the complexities of cancer immune evasion have yielded several other important observations; tumor cells generate foreign neoantigens that can be recognized by host effector $\mathrm{T}$ cells (5). Tumors with a high mutational burden may respond better to immunotherapy, resulting from a diverse genomic landscape driving neoantigen load and favoring immune recognition (6). A T cell-rich or inflamed immunophenotype exists among certain tumors, comprising an interferon $\gamma($ IFN- $\gamma)$ signature and distinct cytokine profile (7) - our own work has shown this among SCCHN tumors (8). While these remain important preliminary observations, they do not currently inform patient selection for checkpoint inhibitor use.

Here we present a clinically annotated cohort of SCCHN patients treated with anti-PD-1/L1 therapies. Targeted massively parallel sequencing and tumor immune profiling results were analyzed to further nominate predictors of checkpoint inhibitor response. 
Table 1. Demographics and clinical characteristics in anti-PD-1/L1 treated patients with SCCHN

\begin{tabular}{|c|c|}
\hline Characteristic & $(\%)^{A} N=126$ \\
\hline Age (median, y) & $57(20-89)$ \\
\hline \multicolumn{2}{|l|}{ Gender } \\
\hline Male & $104(83)$ \\
\hline Female & $22(17)$ \\
\hline \multicolumn{2}{|l|}{ Smoking status } \\
\hline Never or $<10$ packs/year & $60(48)$ \\
\hline Former ( $\geq 10$ packs/year) & $57(45)$ \\
\hline Current & $9(7)$ \\
\hline \multicolumn{2}{|l|}{ ECOG $^{\mathrm{B}}$ performance status } \\
\hline 0 to 1 & 107 (85) \\
\hline 2 or greater & $19(15)$ \\
\hline \multicolumn{2}{|l|}{ Primary site of disease } \\
\hline Oral cavity & $28(22)$ \\
\hline Oropharynx & $56(44)$ \\
\hline Nasopharynx & $9(7)$ \\
\hline Larynx & $14(11)$ \\
\hline Cutaneous & $12(10)$ \\
\hline Unknown & $7(6)$ \\
\hline \multicolumn{2}{|l|}{ Initial staging at diagnosis } \\
\hline Stage I, II & $16(13)$ \\
\hline Stage III, IV & $110(87)$ \\
\hline \multicolumn{2}{|l|}{ Viral status ${ }^{c}$} \\
\hline $\mathrm{HPV}^{+}$ & $50(40)$ \\
\hline $\mathrm{HPV}^{-}$ & $76(60)$ \\
\hline $\mathrm{EBV}^{+}$ & $7(6)$ \\
\hline \multicolumn{2}{|l|}{ Initial treatment regimen } \\
\hline Surgery & $18(14)$ \\
\hline Surgery + radiation & $10(8)$ \\
\hline Surgery + CRT & $21(17)$ \\
\hline Definitive CRT & $56(44)$ \\
\hline Platinum-based & 51 \\
\hline Cetuximab-based & 5 \\
\hline IC + CRT (sequential) & $13(10)$ \\
\hline TPF & 10 \\
\hline $\mathrm{PCC}$ & 3 \\
\hline Chemotherapy & $8(6)$ \\
\hline \multicolumn{2}{|l|}{ Recurrent or metastatic disease } \\
\hline $\begin{array}{l}\text { Locoregional recurrence or } \\
\text { persistent disease }\end{array}$ & $84(67)$ \\
\hline Distant disease $\mathrm{D}^{\mathrm{D}}$ & $42(33)$ \\
\hline
\end{tabular}

${ }^{A}$ Except for age. ${ }^{B}$ Eastern Cooperative Oncology Group. 'HPV status was tested in all oropharyngeal and unknown primaries and assumed to be negative at other sites. ${ }^{D} n$ = 8 patients presented at initial diagnosis with distant disease. SCCHN, squamous cell carcinoma of the head and neck; HPV, human papillomavirus; EBV, EpsteinBarr virus; $C R T$, concurrent chemoradiation; IC, induction chemotherapy; TPF, docetaxel/cisplatin/5-fluorouracil; PCC, paclitaxel/carboplatin/cetuximab.

\section{Results}

Clinical characteristics of the cohort. One hundred and twenty-six patients with SCCHN treated with anti-PD-1/L1 therapy at our institution were included. This cohort was composed mostly of men $(104,83 \%)$ and nearly half were never smokers (Table 1$)$. Oral cavity and oropharyngeal tumors $(84,66 \%)$ were the most common primary site of disease. HPV-associated disease was confirmed in $50(40 \%)$ patients (in 50 of $63,79 \%$ of oropharyngeal or carcinoma of unknown primary [CUP] cases). A majority of patients were treated with some combination of radiation and chemotherapy $(95,75 \%)$, but 31 (25\%) received surgery and/or radiation, or chemotherapy alone for treatment of initial disease. Platinum-based chemotherapy was utilized in most cases. Most patients experienced locoregionally recurrent or persistent disease $(84,67 \%)$, and only $8(6 \%)$ patients presented with distant disease at initial presentation.

Clinical efficacy and tolerability of PD-1/L1 blockade. Six (5\%) complete responses (CRs) and eleven (9\%) partial responses (PRs) were observed (by Response Evaluation Criteria in Solid Tumors [RECIST] version 1.1), with a clinical benefit rate $(\mathrm{CBR}=\mathrm{CR}+\mathrm{PR}+\mathrm{SD}$ [stable disease] $)$ of 39\% (Table 2). Median follow-up time was 8.6 months. Ten patient responses $(8 \%)$ could not be assessed at the time of analysis, as on-treatment imaging was not yet available. Median time to response was 6.2 weeks (range 3-14). There was no difference in the number of responses observed among $\mathrm{HPV}^{+} /$Epstein-Barr virus-positive $\left(\mathrm{EBV}^{+}\right)$and virus-negative patients ( 7 vs. $10, P=0.54$ ). The only significant clinical predictor of response was chemotherapy exposure (as part of initial treatment) prior to anti-PD-1/L1 treatment (hazard ratio [HR] 0.07; 95\% CI, 0.01-0.54; $P=0.01$ ) (Supplemental Table 1; supplemental material available online with this article; https://doi.org/10.1172/ jci.insight.98811DS1). Importantly, there was no difference in response outcomes between the sequenced and immunoprofiled subgroups among the cohort (12 vs. 8 , $P=0.17$ ) (Supplemental Table 2). The rate of disease progression among responders was $18 \%$ ( 3 of 17), with a median duration of response of 7.9 months (range 1-27 or more months).

Fifty-seven deaths occurred with a median overall survival (OS) of 9.0 months (95\% CI, 9.7-11.4) among all patients, with a 1-year OS rate of $38.6 \%$. Among clinical variables, multivariate analysis confirmed an increased risk of death associated with prior smoking (HR 1.57, $P<0.01$ ) and viral tumor status (HR 1.89, $P<0.05$ ) (Supplemental Figure 1). $\mathrm{HPV}^{+} / \mathrm{EBV}^{+}$patients demonstrated improved OS compared with virus-negative patients treated with PD-1/L1 blockade (11 vs. 7 months, HR 0.58 95\% CI, 0.32-1.04; $P=0.02$ ). Those patients treated with prior chemotherapy (with radiation or alone) compared with radiation and/or surgery alone had improved OS (10 vs. 3 months, HR $0.47 ; 95 \% \mathrm{CI}, 0.18-1.22 ; P=0.02$ ), although more $\mathrm{HPV}^{+} / \mathrm{EBV}^{+}$ patients received chemotherapy. Twelve $(10 \%)$ patients experienced grade $3+$ adverse events (using Common Terminology Criteria for Adverse Events [CTCAE]) warranting treatment discontinuation, including subacute thyroiditis (2 patients); pneumonitis (2 patients); gastrointestinal, hepatic, or pancreatic inflammation (5 patients); or acute cervical neck or facial edema within days of receiving a checkpoint inhibitor (3 patients). Seven $(58 \%)$ of those with significant toxicity had evidence of SD or response.

Genomic insights to PD-1/L1 response. Eighty-one (64\%) patients had targeted massively parallel sequencing (OncoPanel) data obtained prior to the initiation of PD-1/L1 blockade (Supplemental Table 3). We first sought to determine total mutational burden (TMB) among the genomic cohort and its impact on outcomes. Normalized TMB ranged from 1.5 to 76.0 mutations/Mb (median 7.6). TMB for the entire cohort was significantly higher among responders compared with nonresponders (median 17.7 vs. 7.1, $P<0.01$ ) (Figure 1). Similarly, in multivariate analysis TMB was significantly associated with improved outcomes (HR 1.94, $P$ $<0.05$ ) (Supplemental Figure 1). When accounting for viral tumor status, patients 
Table 2. Clinical response and outcomes to anti-PD-1/L1 therapy in patients with SCCHN

\begin{tabular}{|c|c|c|c|c|}
\hline & Overall $(N=126)$ & $\mathrm{HPV}^{+} / \mathrm{EBV}^{+}(\mathrm{N}=50)$ & $\mathrm{HPV}^{-} / \mathrm{EBV}^{-}(N=76)$ & $P$ value \\
\hline $\mathrm{CR}$ & $6(5)^{A}$ & $4(8)$ & $2(3)$ & \\
\hline PR & $11(9)$ & $3(6)$ & $8(11)$ & \\
\hline SD & $32(25)$ & $14(28)$ & $18(24)$ & \\
\hline PD & $67(53)$ & $25(50)$ & $42(55)$ & \\
\hline Unevaluable ${ }^{B}$ & $10(8)$ & $4(8)$ & $6(8)$ & \\
\hline Clinical benefit rate ${ }^{c}$ & 49 (39) & $21(42)$ & $28(37)$ & \\
\hline Number of deaths & 57 & 22 & 35 & \\
\hline Median OS (95\% Cl) & $9.0(9.7-11.4)$ & $11.0(3.4-29.2)$ & $7.0(1.4-13.7)$ & HR $0.58(0.32-1.04), P=0.02$ \\
\hline 1 -year OS (95\% CI) & $38.6 \%(27.1 \%-50.3 \%)$ & $42.1 \%(23.8 \%-61.2 \%)$ & $39.3 \%(22.6 \%-53.1 \%)$ & \\
\hline Median follow-up (range) & $8.6(1.3-29.4)$ & & & \\
\hline \multicolumn{5}{|c|}{$\begin{array}{l}\text { A(equals \%). BTreatment ongoing and no interval imaging available at the time of analysis. SCCHN, squamous cell carcinoma of the head and neck; HPV, } \\
\text { human papillomavirus; EBV, Epstein-Barr virus; CR, complete response; PR, partial response; SD, stable disease; PD, progression of disease; OS, overall } \\
\text { survival; HR, hazard ratio. 'Equals CR + PR + SD. }\end{array}$} \\
\hline
\end{tabular}

with non-virally mediated tumors had a higher TMB (8.2 vs. 4.7, $P<0.01)$. Additionally, TMB was significantly higher among $\mathrm{HPV}^{-} / \mathrm{EBV}^{-}$responders (21.3 vs. 8.2, $\left.P<0.01\right)$, but not among $\mathrm{HPV}^{+} / \mathrm{EBV}^{+}$responders (median 8.2 vs. 7.3, $P=0.62$ ). Moreover, TMB correlated with OS among virus-negative patients, in that patients with a TMB greater than 10 had a median OS of 20.0 versus 6.0 months in those with a TMB less than $5(P=0.01)$ - unlike their $\mathrm{HPV}^{+} / \mathrm{EBV}^{+}$counterparts where TMB did not predict OS. TMB was significantly higher among smokers (10.3 vs. $5.3, P=0.01)$ regardless of viral status, but tobacco exposure alone did not predict clinical response to checkpoint blockade $(P=0.62)$ in multivariate analysis.

We next evaluated the tumor mutational landscape based on anti-PD-1/L1 response. Nonexclusive somatic alterations in NOTCH1, TP53, KMT2D, and SMARCA4 were the most commonly reported mutations among the 12 sequenced responders (Figure 2). When comparing the most commonly mutated genes among sequenced responders, NOTCH1 and SMARCA4 mutations were both observed significantly more frequently in comparison with sequenced nonresponders $\left(P=4.6 \times 10^{-5}\right.$ and $4.7 \times 10^{-5}$, respectively; Bonferroni-corrected $P<0.05)$. When we stratified the genomic cohort by viral status, both mutations were noted to occur more frequently only among $\mathrm{HPV}^{-} / \mathrm{EBV}^{-}$responders $\left(P=3.6 \times 10^{-5}\right.$ and $8.2 \times 10^{-5}$, respectively). When considering mechanisms of alteration, in-frame or frameshift events were more frequently observed among sequenced responders (17 of 243 vs. 33 of 868 single-nucleotide variants [SNVs], $P=0.03$ ), and occurred almost always in genes with known tumor suppressor function. Again, this finding was only significant among $\mathrm{HPV}^{-} / \mathrm{EBV}^{-}$responders when accounting for viral tumor status $(P=0.01)$. Supplemental Figure 2 shows the tumor mutational landscape among patients with progressive disease (PD) for comparison.

Among sequenced responders, frequent copy-number alterations included single copy-number losses in $A T M, C B L$, and $S D H D$; as well as low copy gains in ETV5, MECOM, PIK3CA, PRKCI, and $S O X 2$ (Figure 3). However, there was no difference in the fraction of the genome that was copy-number altered between responders and nonresponders $(0.17$ vs. $0.16, P=0.65)$, regardless of viral status (virus positive: 0.15 vs. $0.17, P=0.34$, virus negative: 0.17 vs. $0.16, P=0.51$ ). Of interest, case 1 in Figure 2 had high copy gain and amplification of CD274/PDCD1LG2 at 9p24 (estimated copy count 38 and 62 , respectively) while 6 other patients with a clinical benefit had low copy gains in this gene. Supplemental Figure 3 shows copy-number alterations among nonresponders with PD. No recurrent rearrangements were identified among responders.

We next screened for established mutational signatures (9-11) among the responders. One responder (case 9) had evidence of mismatch-repair (MMR) deficiency. Overall homopolymer insertion and deletion (indel) count - a proposed surrogate for microsatellite instability — ranged from 0 to 3.8 among the genomic cohort, and was significantly elevated among responders $(P<0.01)$, with the highest value occurring in case 9 (3.8 indels), as expected. We also assessed total indel count by counting all nonsynonymous, protein changing, non-SNVs per sample: responders had greater total indel 
A

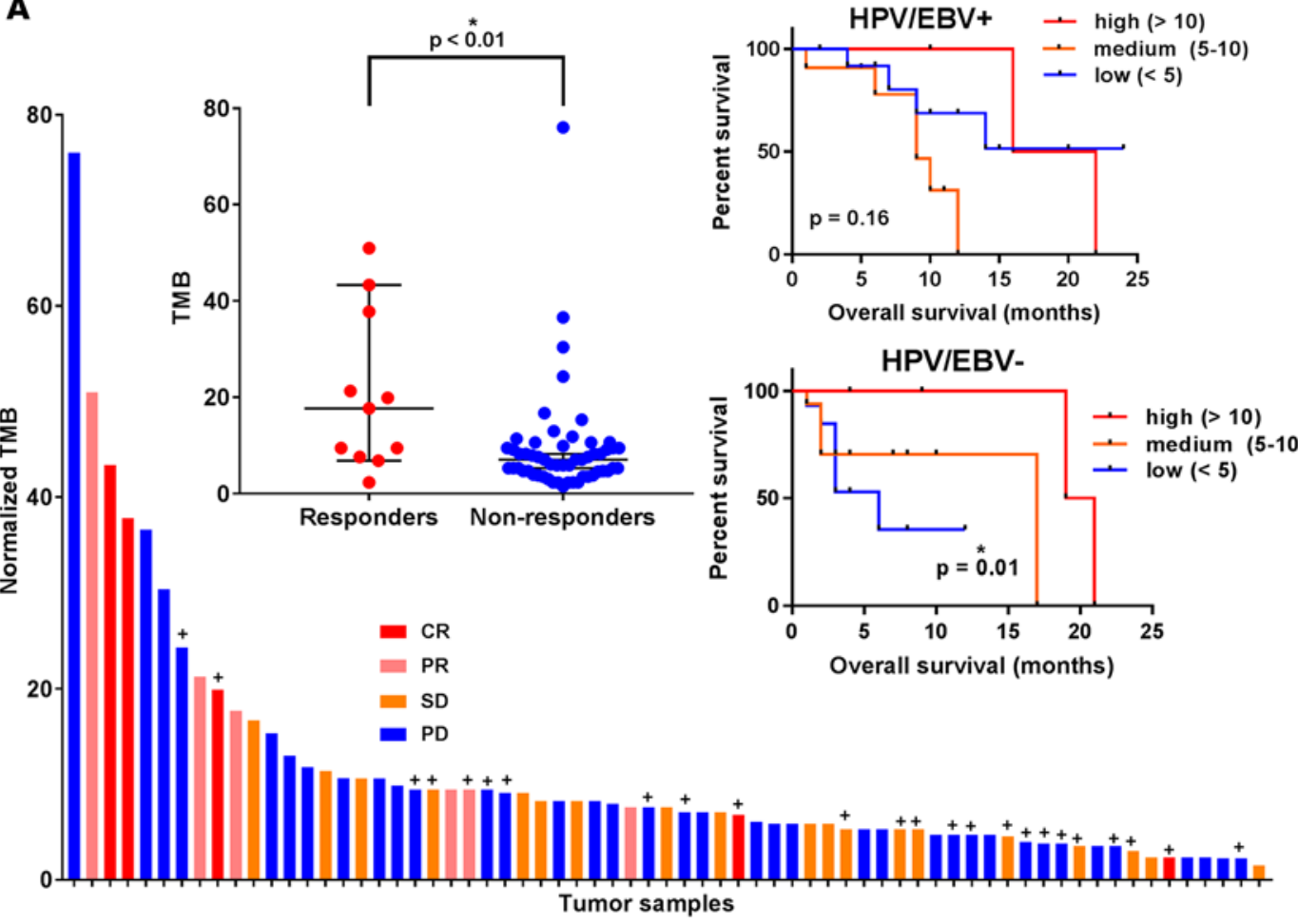

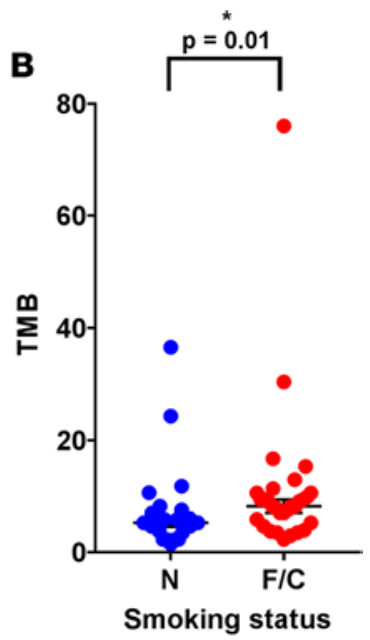

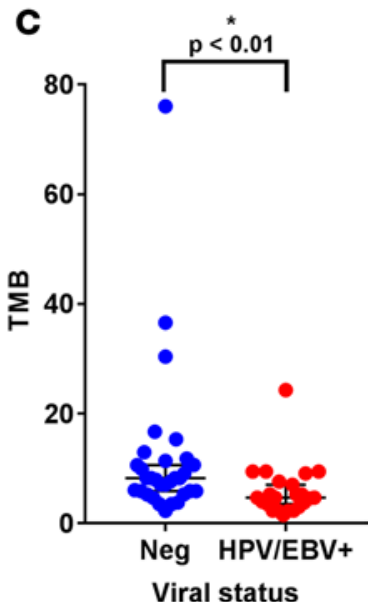

Figure 1. Correlating total mutational burden with response to PD-1/L1 blockade in SCCHN. (A) Normalized total mutational burden (TMB) among tumor samples ( $n=81)$ arranged from highest to lowest according to anti-PD-1/L1 response. A (+) denotes virus-positive disease. Responders show significantly increased TMB compared with nonresponders. HPV-negative patients with higher TMB demonstrate prolonged overall survival (OS). Greater TMB among (B) former or current (F/C) smokers compared with never (N) smokers, and (C) among patients with virus-mediated [HPV or EBV] disease. SCCHN, squamous cell carcinoma of the head and neck; CR, complete response; PR, partial response; SD, stable disease; PD, progressive disease; EBV, Epstein-Barr virus; HPV, human papillomavirus. Horizontal bars show median and $95 \%$ confidence intervals. Mann-Whitney $U$ test, log-rank testing.

counts (median 1.5 vs. $1, P=0.02$ ), but when stratifying by HPV/EBV status, these findings were only significant among $\mathrm{HPV}^{-} / \mathrm{EBV}^{-}$tumors $(P=0.02)$. Among all responders, TMB correlated with total indel count $(R=0.69, P<0.01)$, but again this was only upheld for virus-negative tumors $(R=0.81$, $P<0.01$ ). One responder (case 1 ) had an apolipoprotein B mRNA editing enzyme, catalytic polypeptide-like (APOBEC) signature, and 3 (cases 2, 4 and 12) had an ultraviolet (UV) signature. No responders exhibited a dominant smoking signature. Of the remaining responders, 3 of 7 ( $43 \%$, cases 5 , 7 , and 8) had frameshift or in-frame deletions in at least one tumor suppressor gene (frameshift signature) without dominance of an established mutational signature.

Immune profiling insights to anti-PD-1/L1 response. Among sequenced tumors, $52 \%$ (42 of 81 ) of samples underwent multiparametric flow cytometry and immunohistochemical staining for PD-L1 to facilitate comprehensive immunoprofiling. Flow analysis was pursued if fresh tissue biopsy material was available prior to checkpoint inhibition. Flow data showed a median of $20 \% \mathrm{CD}^{+} \mathrm{T}$ cells (median 371.7 absolute $\mathrm{T}$ cells per sample) and a median $\mathrm{CD} 8^{+} / \mathrm{CD} 4^{+}$ratio of $0.8 . \mathrm{CD}^{+} \mathrm{T}$ cell infiltrates were higher among responders $\left(56.2 \%\right.$ vs. $33.9 \%$ of $\mathrm{CD}^{+} \mathrm{T}$ cells, $\left.P<0.01\right)$ and were an independent predictor of improved outcomes in multivariate analysis (HR 1.91, $P<0.05$ ). They often displayed an activated $\left(64 \%, \mathrm{CD} 38^{+}\right.$or $\left.\mathrm{CD}^{2} 9^{+}\right)$effector memory phenotype $\left(84.4 \%, \mathrm{CD}^{2} 5 \mathrm{RO}^{+} \mathrm{CCR} 7^{-}\right)$(Figure 4$)$. $\mathrm{CD} 8^{+} \mathrm{T}$ cell abundance remained higher among responders regardless of tumor viral status $\left(\mathrm{HPV}^{+} /\right.$ 


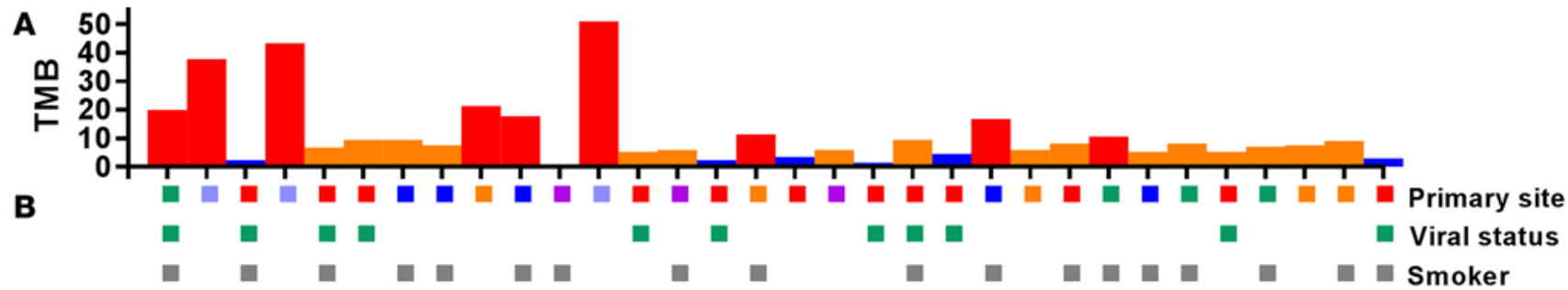

C

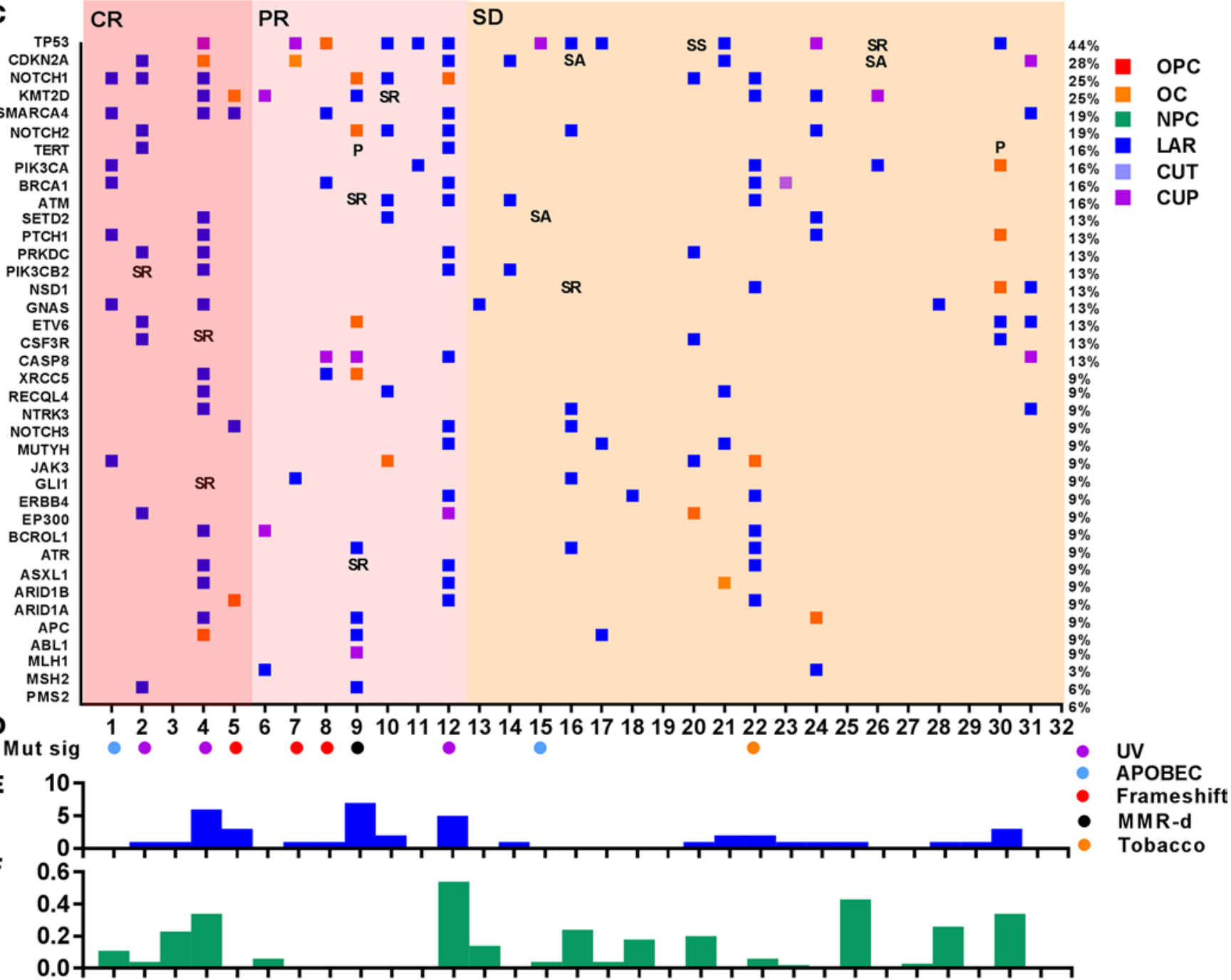

G

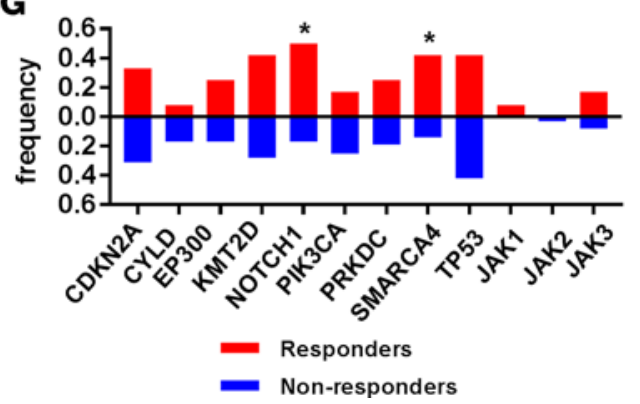

Figure 2. Genomic landscape among anti-PD-1/L1 responders in SCCHN. (A) Genomic mutational landscape among anti-PD-1/L1 responders $(n=12)$ and those with SD ( $n=$ 20) using a targeted next-generation sequencing platform highlighting total mutational burden (TMB) grouped by high (red, > 10 mutations/Mb), medium (orange, 5-10 mutations/Mb), and low (blue, < 5 mutations/Mb). (B) Primary site of disease (key: top right), viral status $\left(\mathrm{EBV}^{+}\right.$or $\mathrm{HPV}^{+}$) and smoking status are shown. (C) The mutational plot shows somatic alterations in order of frequency (highest on top). Somatic mutation key: blue (missense), purple (nonsense), orange (in-frame or frameshift). SA, splice acceptor; SS, splice site; SR, splice region; $\mathrm{P}$, promoter alteration. Only those alterations occurring in 3 or more tumor samples are included in the grid with the exception of genes involved in mismatch repair (MMR). (D) Mutational signatures are displayed (key: lower right). (E) Total indel count (TIC) per tumor sample. (F) Fraction of the genome that is copy-number altered. (G) Proportion of patients with key mutations by response. ${ }^{*} P<0.01$ ( $\chi^{2}$ test, 2-sided). SCCHN, squamous cell carcinoma of the head and neck; UV, ultraviolet; CR, complete response; PR, partial response; SD, stable disease; OPC, oropharynx; OC, oral cavity; NPC, nasopharynx; LAR, larynx, hypopharynx; CUT, cutaneous; CUP, carcinoma of unknown primary; APOBEC, apolipoprotein B mRNA editing enzyme, catalytic polypeptide-like. 


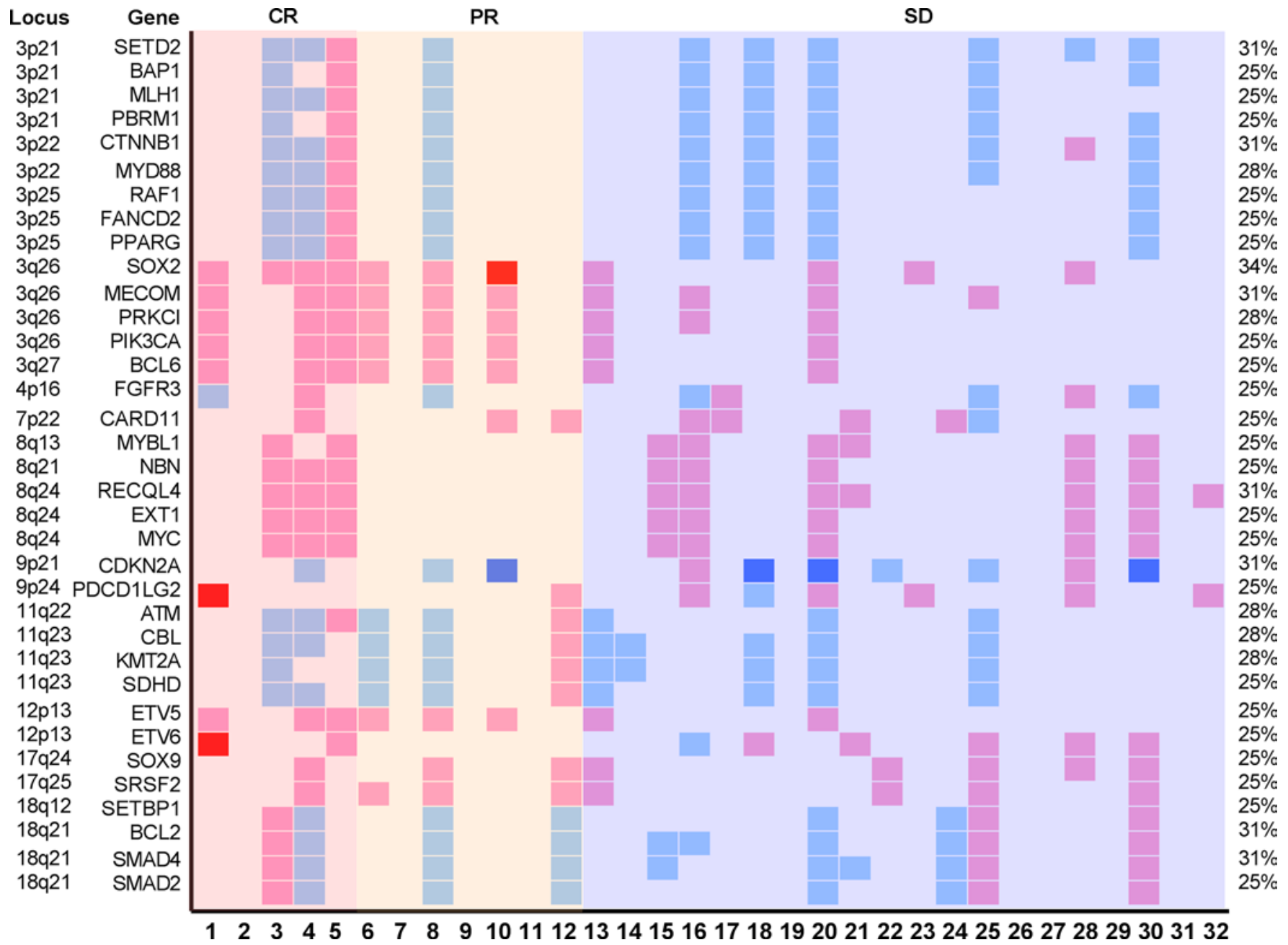

Figure 3. Copy-number alteration events among anti-PD-1/L1 responders in SCCHN. Copy-number variation (CNV) plot showing losses and gains in order of gene loci among those genes with the greatest frequency of alteration among the cohort ( $\geq 8$ events). CNV key: light blue (single copy loss), dark blue (homozygous deletion), pink (low copy gain), red (amplification). SCCHN, squamous cell carcinoma of the head and neck; CR, complete response; PR, partial response; SD, stable disease.

$\mathrm{EBV}^{+}: 63.5 \%$ vs. $33.9 \%, P<0.01$; virus negative: $56.8 \%$ vs. $\left.33.2 \%, P<0.01\right)$. CD8 ${ }^{+} \mathrm{T}$ cell abundance also correlated with TMB among responders $(R=0.91, P<0.01)$ with virus-negative tumors. PD- $1^{+}$ $\mathrm{CD}^{+} \mathrm{T}$ cells were similar among response groups $(21.6 \%$ vs. $18.9 \%, P=\mathrm{NS})$ regardless of $\mathrm{HPV} / \mathrm{EBV}$ status. Monocyte PD-L1 expression was higher among responders $(48.1 \%$ vs. $18.7 \%, P<0.01)$, while PD-L2 expression was similar using flow cytometric analysis. When stratifying by tumor viral status, $\mathrm{PD}-\mathrm{L}^{+}$monocytes were only higher in $\mathrm{HPV}^{+} / \mathrm{EBV}^{+}$tumors $(64.9 \%$ vs. $14.4 \%, P<0.01)$. TIM-3 or LAG-3 coexpression with PD-1 was higher on $\mathrm{CD}^{+}$T cells among nonresponders $(12.4 \%$ vs. $4.4 \%, P$ $=0.03 ; 23.1 \%$ vs. $1.2 \%, P=0.02$, respectively) regardless of tumor viral status.

Among the immunoprofiled cohort, 15 (36\%) cases showed 50\% or higher PD-L1 positivity on tumor cells using immunohistochemistry (IHC), whereas 69\% (29 of 42) had 1\% or higher positivity. Using the $50 \%$ or higher and $1 \%$ or higher cutoffs for PD-L1 yielded $63 \%$ and $88 \%$ of responders with $\mathrm{PD}-\mathrm{L}^{+}$tumors, respectively. Significantly more responders compared with nonresponders $(63 \%$ vs. $29 \%, P=0.03$ ) had PD-L $1^{+}$tumors using the $50 \%$ cutoff, and similarly a PD-L $1^{+}$tumor (using a $\geq 1 \%$ cutoff) was an independent predictor of survival (HR 2.15, $P<0.05$ ) on multivariate analysis. All 4 patients with virus-mediated tumors among the immunoprofiled response group were PD-L1+ by IHC. Tumor IHC and infiltrating monocyte PD-L1 expression by flow correlated $(R=0.87, P<0.01)$ among responders, regardless of $\mathrm{HPV} / \mathrm{EBV}$ status. 

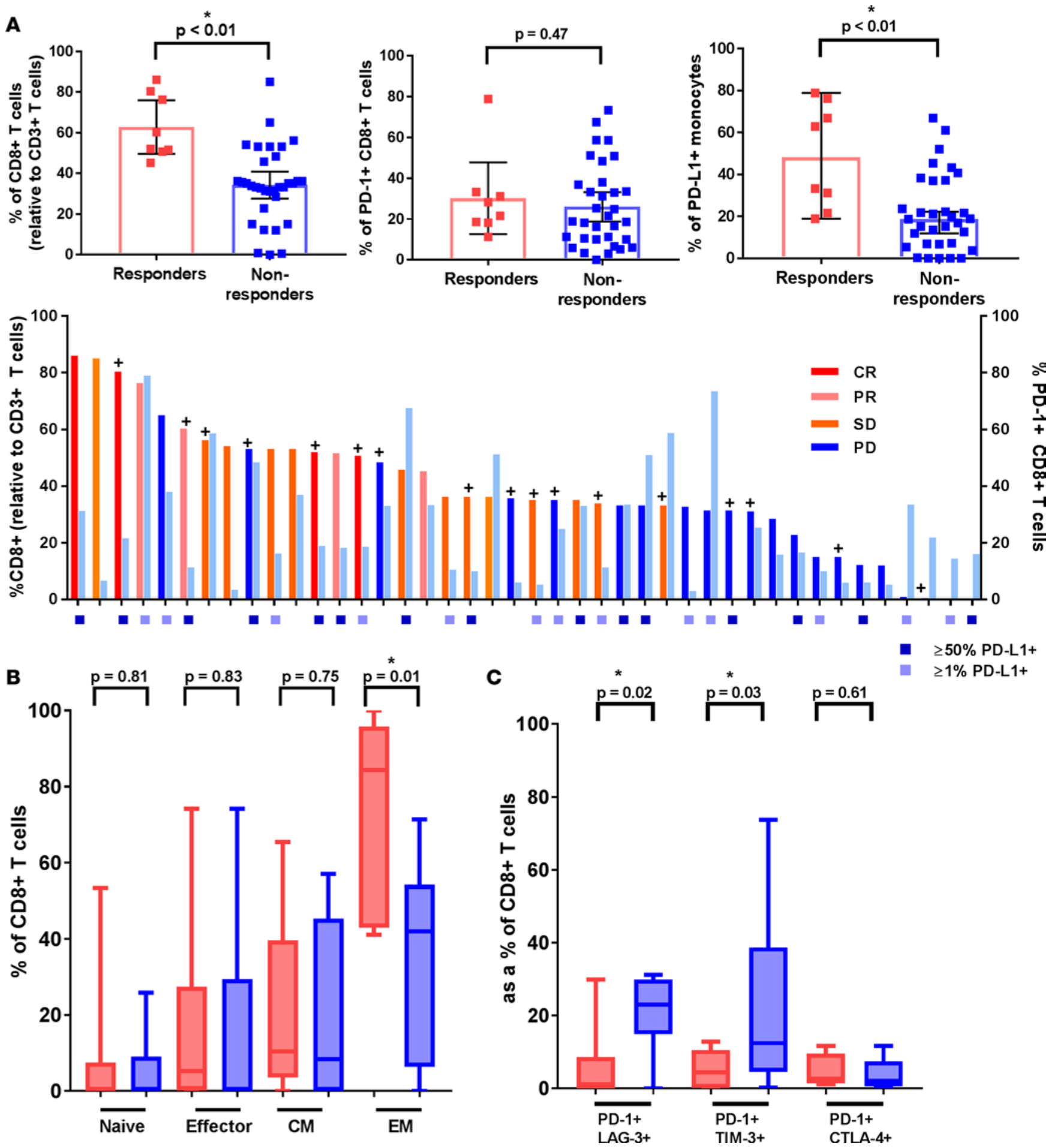

Figure 4. Correlating immunophenotype with response among anti-PD-1/L1 treated patients with SCCHN. (A) Increased tumor CD8 ${ }^{+}$T cell infiltration and PD-L1+ infiltrating monocytes among anti-PD-1/L1 responders. Matched PD-1+ CD8+ T cell component (light blue columns). A (+) indicates a virus-mediated tumor. PD-L1 tumor positivity $(\%)$ by immunohistochemistry $(n=42)$. (B) Immune cell phenotyping shows greater effector memory (EM) CD8 ${ }^{+}$T cells among anti-PD-1/L1 responders $(n=8)$. (C) Immune checkpoint coexpression appears increased among anti-PD-1/L1 nonresponders $(n=34)$. Horizontal bars reflect median and $95 \%$ confidence intervals. ${ }^{*} P<0.05$ determined by Mann-Whitney test, Spearman's $\rho$. SCCHN, squamous cell carcinoma of the head and neck; CM, central memory; CR, complete response; PR, partial response; SD, stable disease; PD, progressive disease. 


\section{Discussion}

We report our institutional experience with anti-PD-1/L1 therapy in SCCHN and show comparable response rates among evaluable patients (17 of $116,15 \%)$ when reviewing recently published trials utilizing PD-1 blockade in advanced SCCHN $(13 \%-18 \%)(2-4,12)$. Some variability in tobacco exposure, HPV status, and anti-PD-1/L1 agent among cohorts explains modest variations in objective response. While the KEYNOTE-12 trial investigating pembrolizumab in advanced SCCHN showed improved response rates in HPV-positive versus -negative patients $(25 \%-32 \%$ vs. $14 \%)$, this has not been observed in all SCCHN trials using PD-1 blockade $(2,4)$. Similar to results from CHECKMATE-141 and KEYNOTE-40 using nivolumab and pembrolizumab in platinum-refractory patients, respectively $(3,13)$, we show a median OS of 9.0 months in anti-PD-1/L1-treated patients, and report a 1-year OS rate of $38.6 \%$. While improved responses have been observed using salvage chemotherapy following anti-PD-1/L1 exposure in non-small cell lung cancer (14), we found platinum-based chemotherapy to be a predictor of anti-PD-1/L1 response in SCCHN. In fact, $82 \%$ of responders in our cohort received prior platinum-based therapy. Interestingly, platinum-based therapies are not often considered immunogenic chemotherapeutic agents (15), but it may be that the addition of concurrent radiotherapy enhances their immunogenic potential (16).

We coupled response with targeted sequencing data and show that TMB predicts checkpoint inhibitor response among virus-negative tumors — in line with observations reported in lung cancer (14). Similarly, prior work has shown that mutational load and an IFN- $\gamma$ gene expression signature appear to predict pembrolizumab response in a virus-negative SCCHN cohort (17). While smokers and patients with virusnegative tumors had higher TMBs in our study, these clinical features alone did not predict anti-PD-1/ $\mathrm{L} 1$ response. In our SCCHN population, we show that patients with virus-negative tumors yielding higher TMBs (>10) had improved OS, which was expected given that TMB correlated with response. Chalmers and colleagues recently described a median of 5 mutations/Mb among SCCHN tumors and $10 \%$ of these tumors had TMB values greater than 20 (18); our results were similar, with a median of 7.6 mutations/ $\mathrm{Mb}$ and $13 \%$ of tumors showing TMB values greater than 20 ( 3 were responders). Virus-associated cancers have been shown to carry lower TMBs (19). Some have suggested that upregulation of virus-specific genes contribute to neoantigen load driving immune checkpoint inhibitor response, rather than TMB (20). We show similar findings in this HPV/EBV-restricted viral population, with median TMB similar between virus-positive patients regardless of response outcomes.

Somatic alterations in several genes with known tumor suppressor function were frequently observed among our virus-negative PD-1/L1 responders: notably NOTCH1 and SMARCA4. NOTCH1 alterations are not uncommon in SCCHN (estimated at 17\%-19\% in advanced disease) (21), but appear significantly further enriched among our nonviral responders. Frequent alterations in SMARCA4 among our virus-negative subgroup were somewhat unexpected, as they are estimated to occur in 5\% of SCCHN tumors (22). SMARCA4 encodes a catalytic subunit of SWF/SNF complexes that acts to regulate gene expression by chromatin remodeling (23). Recent data suggest that EZH2 inhibition may be of clinical utility in cancers harboring mutations in chromatic remodeling genes (24). Of note was the mechanism of alteration in these genes with tumor suppressor function: with many of these mutations resulting from in-frame or frameshift events. Similar to MMR deficiency, these frameshift events may lead to errors in the genomic reading frame that yield an intermediate-to-high mutational load (though this measure is not captured in current standard approaches that report TMB) and ultimately checkpoint inhibitor response. We reported overall homopolymer and total indel counts to quantify highly repetitive regions that could be a surrogate for MMR deficiency (10), which has been shown to yield more somatic alterations and greater immune recognition leading to anti-PD-1 response $(25,26)$. However, among our virus-negative responders, the MMR-deficient signature was only detected in 1 patient. A UV signature and resulting high TMB was observed in 3 responders, which has also been linked to PD-1 response in cutaneous malignancies (27). An APOBEC signature was observed in a patient with EBV-associated disease, which has been described previously (28), and the other virus-positive responders had low to intermediate TMBs. It was surprising that among sequenced patients with a clinical benefit to anti-PD-1/L1 therapy, only 1 (case 22) demonstrated a dominant tobacco signature. Among the remaining sequenced responders, $43 \%$ had somatic frameshift or in-frame events in tumor suppressors (compared with 11 of $63,17 \%$ of the sequenced nonresponders), but again this was significantly observed in only virus-negative tumors. We define this potentially novel mutational signature based on the frequency of frameshift events and lack of an alternative signature. Turajlic and colleagues recently reported the mutagenic potential of small indels, whereby frameshift events can lead to novel open 
reading frames and facilitate generation of a large array of neoantigens with immunogenic potential (29). While renal cell cancers and melanoma had the highest indel proportion in their study of over 5,000 tumor samples, SCCHN was included and had a moderate proportion of indels. They too describe enrichment for tumor suppressor genes among those alterations characterized as frameshift.

The presence of the other commonly mutated genes among our responders $(K M T 2 D, C K D N 2 A$, and $\mathrm{NOTCH} 2$ ) were all observed at similar frequencies regardless of response when accounting for HPV/EBV status, similar to previously described reports characterizing the SCCHN mutational landscape (30). Of note, JAK1 and JAK2 mutations were not associated with resistance to PD-1/L1 blockade in our cohort, as previously reported in melanoma (31), which suggests that different alterations may define immunotherapy resistance among specific tumor types. The lack of epidermal growth factor receptor (EGFR) copy-number gains among the entire cohort was interesting, but could be explained by prior chemoradiotherapy and/or cetuximab exposure in this heavily pretreated group.

Fitting with the observation in other tumor types that a higher TMB generates greater neoantigen potential and yields an abundant $\mathrm{T}$ cell infiltrate (32), we report a correlation between TMB and CD8 ${ }^{+} \mathrm{T}$ cells among virus-negative responders $(R=0.91, P<0.01)$. CD $8^{+} \mathrm{T}$ cell abundance in the current study was high among responders (median above $50 \%$ of all $\mathrm{CD}^{+} \mathrm{T}$ cells) as compared with nonresponders $(P<0.01)$, even among $\mathrm{HPV}^{+} / \mathrm{EBV}^{+}$tumors. Again, virus-specific neoantigens rather than TMB likely facilitate this $\mathrm{T}$ cell expansion. The dominant immunophenotype comprised an activated $\left(\mathrm{CD} 38^{+}\right.$or $\mathrm{CD}^{2} 9^{+}$) population enriched with effector memory $\mathrm{T}$ cells $\left(\mathrm{CD} 45 \mathrm{RO}^{+} \mathrm{CCR} 7^{-}\right)$. These cells exhibit greater effector function and secrete high levels of IFN- $\gamma$ with significant cytotoxic potential (8). Ribas and colleagues have additionally shown that B cells and myeloid-derived suppressor cells are increased in PD-1 blockade responders, and corroborate our findings of an expanded CD8 ${ }^{+}$effector memory $\mathrm{T}$ cell population among responders (33).

Tumor PD-L1 expression was high across our cohort $\left(58.9 \%\right.$ and $72 \%$ for $\mathrm{HPV}^{+} / \mathrm{EBV}^{+}$and negative patients, respectively), consistent with prior reports characterizing SCCHN populations (34). We also show significantly greater tumor PD-L1 expression among responders, particularly when using a 50\% IHC cutoff — in line with several recently published SCCHN trials that have shown similar results (1-3). Additionally, tumor PD-L1 positivity was a predictor of survival among our cohort. Of interest, the abundance of infiltrating monocytes expressing PD-L1 correlated with tumor PD-L1 results among our entire cohort. Some have suggested that PD-L1-expressing tumor-infiltrating myeloid cells are a stronger indicator of prognosis (35), and thus it is fitting that this correlates with tumor PD-L1 expression and therefore response in our study population.

Another important observation was the pattern of checkpoint receptor coexpression among our nonresponders: both LAG-3 and TIM-3 were more often coexpressed with PD- 1 on CD ${ }^{+} \mathrm{T}$ cells $(P=0.02$ and 0.03 , respectively). Several studies including our own have shown variable patterns of checkpoint coexpression among tumor types based on whether they demonstrate an immune-rich phenotype $(8,36)$. However, work in melanoma has shown that partially exhausted or checkpoint-coexpressing $\mathrm{CD}^{+} \mathrm{T}$ cells maintain IFN- $\gamma$ production and can be associated with anti-PD-1 response, although this was observed in CTLA4-coexpressing $\mathrm{T}$ cells (37). Although speculative, higher checkpoint receptor coexpression in our study suggests a mechanism of adaptive immune resistance, whereby inhibition of the PD-1/L1 axis facilitates upregulation of other key checkpoint moieties to promote immune evasion.

Our study has several limitations. While prospective and representative of a large cohort of SCCHN patients, individuals were treated with various single-agent anti-PD-1/L1 therapies. However, among the responders each received single-agent treatment. Some component of survivorship bias should be considered, given that included patients often progressed following first-line chemotherapy to receive anti-PD-1/L1 treatments, therefore affecting survival outcomes. However, our survival data were in line with several recently published prospective trials investigating a similar population $(2-4,12-13)$. Our targeted sequencing platform assesses over 400 genes and 60 intronic regions with importance in cancer, but does not capture all structural rearrangements or epigenetic events; thus, additional genomic events may be contributing to response that we cannot discern. A targeted sequencing platform can be somewhat limited in its ability to detect mutational signatures, and whole-genome sequencing might shed more light on these genomic patterns; additionally, matched peripheral blood controls were not available. We acknowledge the low number of responders with available fresh tissue for flow cytometric analysis, particularly since it limits the power of statistical comparison when stratifying tumors by viral status. Nonetheless, we show that virus-negative SCCHN anti-PD-1/ L1 responders often harbor in-frame or frameshift alterations arising among specific tumor suppressor genes. 
These responders have a greater TMB and neoantigen potential thought to drive effector $\mathrm{T}$ cell response to overcome tumor immune evasion via the PD-1/L1 axis when exposed to anti-PD-1/L1 therapy, suggesting an important mutational signature predictive of response in some patients. While further validation is warranted, these observations provide potentially novel insights regarding the genomic determinants of immunotherapy response in SCCHN.

\section{Methods}

Study participants and cohorts. One-hundred and twenty-six patients with biopsy-proven SCCHN who received anti-PD-1/L1 treatment at our institution from June 2014 to August 2017 were identified prospectively following institutional review board approval. SCCHN arising at all mucosal subsites regardless of viral status, cutaneous SCCHN and CUP of the head and neck were also included. Of these, 81 patients had fresh or archival tumor material available for targeted sequencing prior to receiving anti-PD-1/L1 treatment. Of the 81 patients sequenced, 42 consented prior to biopsy confirmation of recurrent, metastatic disease to facilitate fresh tumor sample acquisition for flow cytometric analysis. These 42 patients also had IHC performed on formalin-fixed, paraffin-embedded (FFPE) tumor samples to determine tumor PD-L1 status. Patient demographics, clinicopathologic features, and treatment outcomes were recorded. HPV status was reported if assessed (testing is standard for all oropharyngeal cancers and CUP of the head and neck at our institution) using p16 immunoreactivity, followed by confirmatory polymerase chain reaction (PCR) testing. EBVencoded RNA (EBER) IHC was used to confirm EBV positivity in all nasopharyngeal carcinomas included.

Treatment efficacy and outcomes. We defined responders as those participants with radiologic evidence of disease improvement following anti-PD-1/L1 exposure. Responders included patients with tumor target lesions that met RECIST 1.1 criteria for a CR ( $>99 \%$ reduction in the target lesions) or a PR ( $\geq 30 \%$ reduction in target lesions). Nonresponders were defined as those patients with tumor target lesions that met criteria for progressive ( $\geq 20 \%$ increase in the target lesions) or stable disease (not fitting either a PR or PD category). Patients were labeled unevaluable if no initial restaging imaging was available for review at the time of analysis. Efficacy was reported as the best overall response to treatment using the RECIST 1.1 categories determined from investigator-reported data. OS was determined from the date of initiation of PD-1/L1 therapy to death from any cause, otherwise this was censored at date of last follow-up. The clinical benefit rate $(C B R=C R+P R+S D)$ was assessed by accounting for best overall response as evaluated by radiologic imaging at least 3 weeks after initial PD-1/L1 therapy dosing. Duration of response was defined as time from documentation of tumor response to disease progression.

Targeted massively parallel sequencing. All sequenced patients separately consented to our institutional Cancer Research Study (38). Of the 81 tumor samples available for sequencing, 34 (42\%) were obtained from a site of locoregionally recurrent or persistent disease (24 fresh tissue samples, 10 archival), whereas 25 (31\%) were obtained from metastatic sites (18 fresh, 7 archival). The remaining samples (22, 27\%) were archival material from the initial primary tumor. Testing was performed in a CLIA-certified laboratory. For FFPE and fresh-frozen tumors, hematoxylin-eosin-stained slides were prepared and reviewed by a pathologist to identify areas of greater than $20 \%$ tumor for molecular analysis. DNA was isolated using standard extraction methods with a commercial kit (Qiagen) and quantified using Qubit dsDNA detection (Invitrogen). Between 50 and 200 ng tumor DNA was ultrasonically fragmented (Covaris), size-selected, and quantified. Dual-indexed sequencing libraries were prepared from $50 \mathrm{ng}$ fragmented DNA using KAPA HTP library preparation kits (Roche). Libraries were prepared from equimolar amounts of samples and hybridized to OncoPanel, a custom biotinylated RNA bait set (Agilent SureSelect) targeting the full coding regions of 300 genes plus selected intronic regions of 35 genes (OncoPanel version 2; ref. 39); or targeting 447 genes and selected intronic regions of 60 genes (OncoPanel version 3) (genes and introns included in the most updated Oncopanel version 3 are shown in Supplemental Table 4). Dyna MyOne Streptavidin T1 magnetic beads (Invitrogen) were added to capture biotinylated baits and captured libraries. An external magnetic field was then applied and the supernatant containing unbound DNA was discarded. After several washes, the captured libraries were PCR-enriched for 10 cycles using adapter-specific primers, size-selected, and prepared for sequencing. Sequencing was performed using an Illumina HiSeq 2500 with $2 \times 100$ paired-end reads.

Sequencing was performed to an attempted coverage of $300 \times$ per sample. For the cases in this study, the mean coverage was $318 \times$ unique, high-quality, mapped reads per sample (range $80 \times$ to $640 \times$; $50 \times$ minimum required to pass). Samples with a mean target coverage of less than $50 \times$ were failed and excluded from further analysis. The mean percentage target bases greater than 30× was $98.46 \%$ (range $91.5 \%-99.6 \%$ ). 
The raw genomic data (vcf files) are included in a publically available database as per our institution's involvement in AACR Project GENIE. Users can access the data directly via cbioportal (http://www. cbioportal.org/genie), or download the data directly from Sage Bionetworks (https://www.synapse.org).

Data were analyzed by an internally developed bioinformatics pipeline composed of reconfigured publically available tools and internally developed algorithms (RobustCNV, Anotate\&Phase, BreaKmer; ref. $40)$ as described previously $(38,39)$. Briefly, pooled sample reads were deconvoluted and sorted using the Picard tools. Reads were aligned to the reference sequence b37 edition from the Human Genome Reference Consortium, using BWA. Duplicate reads were identified and removed using Picard (version 1.13.0). The alignments were further refined using the Genome Analysis Toolkit (GATK, version 3.3.0) for localized realignment around indel sites and recalibration of the quality scores. Mutation analysis for SNVs was performed using MuTect v. 10.27200 and annotated by Variant Effect Predictor (VEP) v79. Insertions and deletions were called using SomaticIndelDetector (v1.65). Paired germline samples were not sequenced: for each sequencing run, non-neoplastic FFPE blood samples were included as matched controls if available. Variants identified in these control samples due to sequencing artifacts were filtered. Because tumor tissues were tested without a paired normal from individual patients, additional informatics steps were taken to identify common single nucleotide polymorphisms (SNPs): any SNP present at greater than $0.1 \%$ in Exome Variant Server, NHLBI GO Exome Sequencing Project (ESP) (URL: http://evs.gs.washington.edu/EVS/ accessed May 30, 2013) or present in dbSNP was filtered; however, variants also present at least twice in COSMIC were rescued for manual review. For copy number analysis, a custom internal R-based tool (RobustCNV) was used to calculate the fractional coverage of specified genomic intervals compared with the median fractional coverage obtained in a panel of 152 FFPE non-neoplastic samples. Structural variant analysis was performed using BreaKmer (40) followed by review of reads in integrative genomics viewer (IGV).

Technical validation experiments were performed to determine precision, accuracy, sensitivity, specificity, and limit of detection, using samples that had existing genomic characterization using an orthogonal clinical test (e.g., OncoMap, pyrosequencing, Sanger sequencing, PCR/electrophoresis, real-time PCR, aCGH array, karyotype or FISH). One hundred twenty samples with known somatic mutations, indels, copy number alterations, and/or rearrangements were selected. Assay validation has been described previously (39); in summary, sensitivity and specificity for SNVs was 97.8\% (95\% CI: 86.5\%-99.9\%) and 100\% (95\% CI: 99.9\%-100\%), respectively; for indels the test was $97.7 \%$ sensitive (95\% CI: $86.5 \%-99.9 \%$ ) and $100 \%$ specific (95\% CI: $99.9 \%-100 \%)$. For copy number changes the assay was $93 \%$ sensitive $(95 \%$ CI: 87.1\%-96.4\%) and 97.6\% specific (95\% CI: 96.1\%-98.5\%). For rearrangement detection, OncoPanel was $74 \%$ sensitive (95\% CI: $53.4 \%-88.1 \%$ ) and $100 \%$ specific (95\% CI: $69.9 \%-100 \%)$.

$\mathrm{TMB}$ was calculated by determining the number of nonsynonymous somatic mutations that occur per megabase of exonic sequence data across all genes on the panel.

MMR pathway status was evaluated by determining the number of small insertion/deletion events that occur in homopolymer regions within exonic sequence data across all genes on the panel, using an extension of a method previously developed in our laboratory (10). Tumors with an elevated number of such events are classified as MMR deficient, while tumors with a low burden of such events are classified as MMR proficient. The sensitivity and specificity of this approach, using 337 tumors with known (IHC or PCR) MMR status as reference, is $97.9 \%$ and $98.0 \%$, respectively. For tumors with 16 or more mutations, additional mutational signature (Hancock) analysis is performed based upon the pattern of nucleotide substitutions. Mutational signatures that can be detected using this approach include those associated with DNA damage due to ultraviolet light (UVA) exposure, tobacco smoke exposure, prior treatment with alkylating agents (including temozolomide), impaired POLE DNA polymerase function, and APOBEC enzyme dysregulation. Hancock is based on previously published signatures derived from whole-exome sequencing data (9) and was refined by training on targeted exome sequencing data (11). The reported mutational patterns reflect those observed in vitro following exposure to relevant mutagens. The presence of these signatures was further validated against clinicopathologic features in 738 OncoPanel samples including (a) origin at a sun-exposed site, (b) smoking history, (c) prior treatment with temozolomide, (d) concurrent POLE hotspot mutation, or (e) MMR deficiency as detected by OncoPanel. Mutational signatures used in this study had sensitivity ranges from $58 \%-82 \%$ and specificity ranges from $80 \%$ to greater than $99 \%$ relative to matched clinical features. Signatures are interpreted by a pathologist as an observed pattern consistent with the appropriate pathologic mechanism, rather than a definitive assertion.

Tumor immune profiling. At the time of biopsy or surgical resection, fresh tissue samples from an available subset of patients were placed in RPMI-1640 with 10\% fetal bovine serum (FBS). Tumor was then 
confirmed by hematoxylin and eosin staining. A non-fixed cell suspension was prepared for staining with fluorescently conjugated antibody cocktails, as previously described (41). Single-cell suspensions were stained using mouse anti-human surface antibodies against an array of cluster differentiation (CD) and immune checkpoint receptors or ligands to comprehensively characterize tumor immune cell phenotype (8). Cells were fixed and analyzed within 24 hours on a BD Fortessa cell analyzer with FACSDiva software v8.0.1 (BD Biosciences) and analyzed using FlowJo software v10.

IHC using a rabbit anti-PD-L1 monoclonal antibody (PD-L1 rabbit mAb 13684, Cell Signaling Technology, Inc. [CST]) was performed on 4- $\mu$ m-thick sections of FFPE tissue blocks using standard antigen retrieval methods (CC1 buffer, $\mathrm{pH} 8.0$, 950-124, Ventana). Slide sections were prepared and exposed to anti-PD-L1 (CST) at 1:200 for 40 minutes at room temperature. PowerVision Poly-AP Anti-Rabbit IgG (Leica) with Permanent Red was used for antibody detection. Expression of PD-L1 on tumor cells was scored positive if 1 or higher or $50 \%$ or higher based on membranous staining intensity.

Statistics. Pearson's $\chi^{2}$ test was used to compare categorical data for the likelihood of observed differences with regards to clinical response based on HPV status, and the presence of individual mutations among response groups. Binary logistic regression analysis was performed to assess clinical predictors of response. Multivariate analysis utilized the Cox proportional hazards model (only if $n \geq 15$ patients were available in each subgroup) and proportional hazards assumption testing was verified. A Mann-Whitney test to compare ranks (or Kruskal-Wallis test or 1-way ANOVA on ranks for multiple comparisons) was used to analyze continuous genomic data among subgroups. Spearman's $\rho$ was used to evaluate correlation. All statistical tests used a significance cutoff of less than 0.05 and were 2-sided. Bonferroni's correction, $(\alpha=$ $[0.05 / k])$, was applied to adjust the $\alpha$ value, accounting for multiple testing among the genomic subgroups. Kaplan-Meier statistics were applied using log-rank testing to evaluate outcome data. Data were analyzed using Stata/IC (version 14.2).

Study approval. Written informed consent for existing institutional review board-approved protocols was received from all participants prior to inclusion in the study.

\section{Author contributions}

GJH, RU, LEM, and RH conceived and designed the project. GJH, PL, MC, NGC, JDS, JHL, and LEM conducted experiments and acquired data. GJH, FCK, PS, AF, RU, and LEM analyzed and interpreted the data. GJH and LEM drafted the manuscript. All authors reviewed and revised the manuscript.

\section{Acknowledgments}

We thank Profile at Brigham and Women's Hospital and Dana-Farber Cancer Institute (DFCI), and the Center for Cancer Genome Discovery (CCGD) at the DFCI for their assistance in analyzing the sequencing data. We are grateful to our patients for their participation in this research.

Address correspondence to: Glenn J. Hanna, Instructor in Medicine, Harvard Medical School, Department of Medical Oncology, Center for Head \& Neck Oncology, Dana-Farber Cancer Institute, 450 Brookline Avenue, Boston, Massachusetts 02215, USA. Phone: 617.901.3090; Email: glenn_hanna@dfci.harvard.edu.

1. Ferris RL. Immunology and immunotherapy of head and neck cancer. J Clin Oncol. 2015;33(29):3293-3304

2. Chow LQM, et al. Antitumor activity of pembrolizumab in biomarker-unselected patients with recurrent and/or metastatic head and neck squamous cell carcinoma: results from the phase Ib KEYNOTE-012 expansion cohort. J Clin Oncol. 2016;34(32):3838-3845.

3. Ferris RL, et al. Nivolumab for recurrent squamous-cell carcinoma of the head and neck. $N$ Engl J Med. 2016;375(19):1856-1867.

4. Seiwert TY, et al. Safety and clinical activity of pembrolizumab for treatment of recurrent or metastatic squamous cell carcinoma of the head and neck (KEYNOTE-012): an open-label, multicentre, phase 1b trial. Lancet Oncol. 2016;17(7):956-965.

5. Verdegaal EM, et al. Neoantigen landscape dynamics during human melanoma-T cell interactions. Nature. 2016;536(7614):91-95.

6. Rizvi NA, et al. Cancer immunology. Mutational landscape determines sensitivity to PD-1 blockade in non-small cell lung cancer. Science. 2015;348(6230):124-128.

7. Gajewski TF, Schreiber H, Fu YX. Innate and adaptive immune cells in the tumor microenvironment. Nat Immunol. 2013;14(10):1014-1022.

8. Hanna GJ, et al. Defining an inflamed tumor immunophenotype in recurrent, metastatic squamous cell carcinoma of the head and neck. Oral Oncol. 2017;67:61-69.

9. Alexandrov LB, et al. Signatures of mutational processes in human cancer. Nature. 2013;500(7463):415-421. 
10. Nowak JA, et al. Detection of mismatch repair deficiency and microsatellite instability in colorectal adenocarcinoma by targeted next-generation sequencing. J Mol Diagn. 2017;19(1):84-91.

11. Zehir A, et al. Mutational landscape of metastatic cancer revealed from prospective clinical sequencing of 10,000 patients. Nat Med. 2017;23(6):703-713.

12. Bauml J, et al. Pembrolizumab for platinum- and cetuximab-refractory head and neck cancer: results from a single-arm, phase II study. J Clin Oncol. 2017;35(14):1542-1549.

13. Cohen EE, et al. Pembrolizumab (pembro) vs standard of care (SOC) for recurrent or metastatic head and neck squamous cell carcinoma (R/M HNSCC): Phase 3 KEYNOTE-040 trial. Ann Oncol. 2017;28(supp1_5):v605-v649.

14. Leger PD, Rothschild S, Castellanos E, Pillai RN, York SJ, Horn L. Response to salvage chemotherapy following exposure to immune checkpoint inhibitors in patients with non-small cell lung cancer. J Clin Oncol. 2017;35(15_suppl):9084-9084.

15. Pfirschke C, et al. Immunogenic chemotherapy sensitizes tumors to checkpoint blockade therapy. Immunity. 2016;44(2):343-354.

16. Sridharan V, et al. Definitive chemoradiation alters the immunologic landscape and immune checkpoints in head and neck cancer. Br J Cancer. 2016;115(2):252-260.

17. Haddad RI, et al. Genomic determinants of response to pembrolizumab in head and neck squamous cell carcinoma(HNSCC) J Clin Oncol. 2017;35(15_suppl):6009-6009.

18. Chalmers ZR, et al. Analysis of 100,000 human cancer genomes reveals the landscape of tumor mutational burden. Genome Med. 2017;9(1):34.

19. Goh G, et al. Mutational landscape of MCPyV-positive and MCPyV-negative Merkel cell carcinomas with implications for immunotherapy. Oncotarget. 2016;7(3):3403-3415.

20. Swanton C, McGranahan N, Starrett GJ, Harris RS. APOBEC enzymes: mutagenic fuel for cancer evolution and heterogeneity. Cancer Discov. 2015;5(7):704-712.

21. Morris LG, et al. The molecular landscape of recurrent and metastatic head and neck cancers: insights from a precision oncology sequencing platform. JAMA Oncol. 2017;3(2):244-255.

22. Stransky N, et al. The mutational landscape of head and neck squamous cell carcinoma. Science. 2011;333(6046):1157-1160

23. Huang M, Qian F, Hu Y, Ang C, Li Z, Wen Z. Chromatin-remodelling factor BRG1 selectively activates a subset of interferonalpha-inducible genes. Nat Cell Biol. 2002;4(10):774-781.

24. Kim KH, Roberts CW. Targeting EZH2 in cancer. Nat Med. 2016;22(2):128-134.

25. Le DT, et al. Mismatch repair deficiency predicts response of solid tumors to PD-1 blockade. Science. 2017;357(6349):409-413.

26. Overman MJ, et al. Nivolumab in patients with metastatic DNA mismatch repair-deficient or microsatellite instability-high colorectal cancer (CheckMate 142): an open-label, multicentre, phase 2 study. Lancet Oncol. 2017;18(9):1182-1191.

27. Rajkumar S, Watson IR. Molecular characterisation of cutaneous melanoma: creating a framework for targeted and immune therapies. Br J Cancer. 2016;115(2):145-155.

28. $\mathrm{Hu} \mathrm{H}$, et al. Epstein-Barr virus infection of mammary epithelial cells promotes malignant transformation. EBioMedicine. 2016;9:148-160

29. Turajlic S, et al. Insertion-and-deletion-derived tumour-specific neoantigens and the immunogenic phenotype: a pan-cancer analysis. Lancet Oncol. 2017;18(8):1009-1021.

30. Riaz N, Morris LG, Lee W, Chan TA. Unraveling the molecular genetics of head and neck cancer through genome-wide approaches. Genes Dis. 2014;1(1):75-86.

31. Zaretsky JM, et al. Mutations associated with acquired resistance to PD-1 blockade in melanoma. $N$ Engl J Med. 2016;375(9):819-829.

32. Brown SD, Warren RL, Gibb EA, et al. Neo-antigens predicted by tumor genome meta-analysis correlate with increased patient survival. Genome Res. 2014;24(5):743-750.

33. Ribas A, et al. PD-1 blockade expands intratumoral memory T cells. Cancer Immunol Res. 2016;4(3):194-203.

34. Hartley G, Regan D, Guth A, Dow S. Regulation of PD-L1 expression on murine tumor-associated monocytes and macrophages by locally produced TNF- $\alpha$. Cancer Immunol Immunother. 2017;66(4):523-535.

35. Scognamiglio T, Chen YT. Beyond the percentages of PD-L1-positive tumor cells: induced versus constitutive PD-L1 expression in primary and metastatic head and neck squamous cell carcinoma [published online ahead of print September 25, 2017]. Head Neck Pathol. https://doi.org/10.1007/s12105-017-0857-3.

36. Lizotte $\mathrm{PH}$, et al. Multiparametric profiling of non-small-cell lung cancers reveals distinct immunophenotypes. JCI Insight. 2016;1(14):e89014.

37. Daud AI, et al. Tumor immune profiling predicts response to anti-PD-1 therapy in human melanoma. J Clin Invest. 2016;126(9):3447-3452.

38. Sholl LM, et al. Institutional implementation of clinical tumor profiling on an unselected cancer population. JCI Insight. 2016;1(19):e87062.

39. Garcia EP, et al. Validation of OncoPanel: A targeted next-generation sequencing assay for the detection of somatic variants in cancer. Arch Pathol Lab Med. 2017;141(6):751-758.

40. Abo RP, et al. BreaKmer: detection of structural variation in targeted massively parallel sequencing data using kmers. Nucleic Acids Res. 2015;43(3):e19.

41. Awad MM, et al. Cytotoxic T cells in PD-L1-positive malignant pleural mesotheliomas are counterbalanced by distinct immunosuppressive factors. Cancer Immunol Res. 2016;4(12):1038-1048. 